(W)

Check for

Cite as

Nano-Micro Lett.

(2022) 14:62

Received: 29 October 2021

Accepted: 6 January 2022

Published online: 15 February 2022

(C) The Author(s) 2022

\section{Bioinspired Adaptive, Elastic, and Conductive Graphene Structured Thin-Films Achieving High-Efficiency Underwater Detection and Vibration Perception}

\author{
Qiling Wang ${ }^{1,2}$, Peng Xiao ${ }^{1,2}$, Wei Zhou ${ }^{1,2}$, Yun Liang ${ }^{1,2}$, Guangqiang Yin $^{1,2}$, \\ Qiu Yang ${ }^{3}$, Shiao-Wei Kuo ${ }^{4}$, Tao Chen ${ }^{1,2} \bowtie$
}

\title{
HIGHLIGHTS
}

- The lateral-line-like underwater mechanical sensor (LUMS) realizes the imitation of the structure and function of the lateral line.

- The detection range of water depth can be controlled by adjusting the size of the graphene/Ecoflex Janus film on LUMS. The maximum measured depth is $1.8 \mathrm{~m}$.

- Similar to the fish, the mechanical stimuli from land and water can be sensitively captured by LUMS in real time.

\begin{abstract}
Underwater exploration has been an attractive topic for understanding the very nature of the lakes and even deep oceans. In recent years, extensive efforts have been devoted to developing functional materials and their integrated devices for underwater information capturing. However, there still remains a great challenge for water depth detection and vibration monitoring in a high-efficient, controllable, and scalable way. Inspired by the lateral line of fish that can sensitively sense the water depth and environmental stimuli, an ultrathin, elastic, and adaptive underwater sensor based on Ecoflex matrix with embedded assembled graphene sheets is fabricated. The graphene structured thin film is endowed with favourable adaptive and morphable features, which can conformally adhere to the structural surface and transform to a bulged state driven by water pressure. Owing to the introduction of the graphene-based layer, the integrated sens-
\end{abstract}

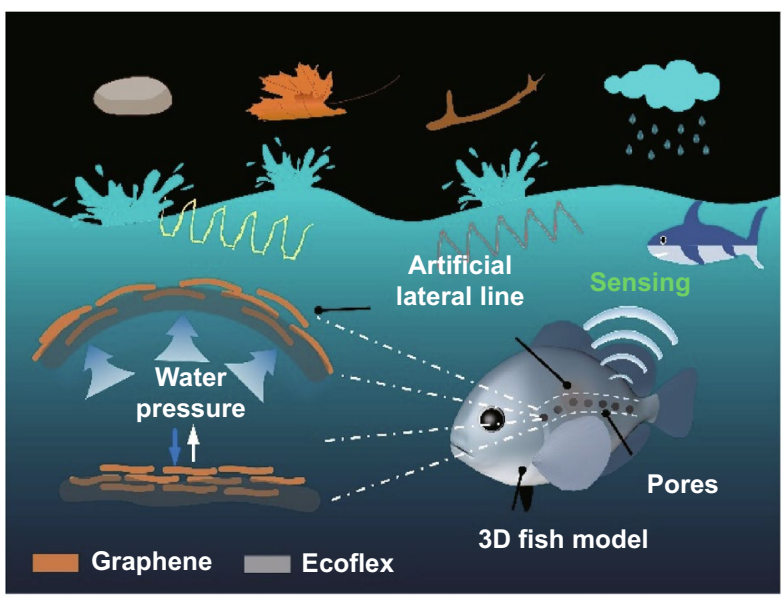
ing system can actively detect the water depth with a wide range of 0.3-1.8 m. Furthermore, similar to the fish, the mechanical stimuli from land (e.g. knocking, stomping) and water (e.g. wind blowing, raining, fishing) can also be sensitively captured in real time. This graphene structured thin-film system is expected to demonstrate significant potentials in underwater monitoring, communication, and risk avoidance.

KEYWORDS Janus film; Water depth detection; Vibration perception

Qiling Wang and Peng Xiao contributed equally to this work.

$\bowtie$ Peng Xiao, xiaopeng@nimte.ac.cn; Tao Chen, tao.chen@nimte.ac.cn

1 Key Laboratory of Marine Materials and Related Technologies, Zhejiang Key Laboratory of Marine Materials and Protective Technologies, Ningbo Institute of Materials Technology and Engineering, Chinese Academy of Sciences, Zhongguan West Road 1219, Ningbo 315201, People's Republic of China

2 School of Chemical Sciences, University of Chinese Academy of Sciences, 19A Yuquan Road, Beijing 100049, People's Republic of China

3 Ningbo New Material Testing and Evaluation Center Co., Ltd, Ningbo 315000, People's Republic of China

4 Department of Material and Optoelectronic Science, Center of Crystal Research, National Sun Yat-Sen University, Kaohsiung 804, Taiwan, People's Republic of China 


\section{Introduction}

Exploration in extreme environments, especially underwater monitoring and communication, is of great significance to understand the unknown and/or unmapped underwater world. Precisely and stably capturing underwater signal of depth and dramatic/ tiny vibration can provide abundant and essential information for underwater forewarning, creature tracking, and environmental considerations [1-6]. To date, extensive efforts have been devoted to developing underwater sensing materials and their integrated devices [7-12]. Soft materials featured with anti-corrosion and flexibility are considered as a promising candidate, enabling piezoresistive [13-15], capacitive [16, 17] and piezoelectric [7, 18] mechanism for underwater sensing. Since the water environment may adversely affect the conductivity of the sensors, some strategies have been developed to tackle this problem, including superhydrophobic sealing [19-23], and polymer encapsulation [24-26]. Among them, the superhydrophobic sealing method heavily relies on the micro-nano-structures $[15,19]$, where the long-term stability may experience unfavourable wettability failure. And the encapsulation one may lose sensitivity of devices. In addition, eye-catching forms of materials and integrated devices may be easily attacked by underwater creatures, which should also be considered. Thus, the design of underwater sensors with flexible, environmentally stable and imperceptible properties for broad water depth detection and tiny vibration perception still remains a challenge in one integrated system.

In nature, fish can actively sense the external environment (e.g. water depth and mechanical vibration) and further adapt their behaviours to the surroundings. In the fish sensing systems, the lateral line has played vital roles in perceiving the external water pressure, flow movement and diverse vibrations (Fig. 1a-c). In water, the pressure change can go through the pores on the scales to the lateral line canal, which can act on the nerves for real-time and high-sensitive underwater sensing (Fig. 1d) [27-31]. Inspired by the fish sensing system, herein, we developed an artificial lateral line system enabled by a flexible and imperceptible thin-film in a self-supported state to simultaneously perceive wide-range underwater depth and large/tiny vibration. To achieve stable underwater sensing, a Janus film structure was designed and further integrated as a self-supported sensor with Ecoflex layer exposed to water. As a proof of concept, an artificial fish lateral line system was constructed to imitate the underwater sensing functions of fish. Owing to the ultrathin and elastic features, the fabricated film-based sensor can experience a remarkable deformation to achieve reversible resistance change for real-time water depth detection with a maximum depth of $1.8 \mathrm{~m}$. Moreover, similar to fish, it can sensitively perceive the tiny vibration above and below the water surface, such as wind blowing, raining, underwater creatures' attacking, etc. The novel and simple design of this work is expected to demonstrate significant potentials in applications of underwater monitoring, communication and rescue.

\section{Materials and Methods}

\subsection{Materials}

Graphene slurry (5 wt\%) with surface defects (Supplementary Fig. S1) obtained by mechanical exfoliation was acquired from Ningbo Morsh Technology Co., Ltd, and the size range of graphene flakes was $\sim 2.5-7 \mu \mathrm{m}$ (Supplementary Fig. S2). Owing to the multi-layered structure, the thickness of graphene flakes was $\sim 10 \mathrm{~nm}$ (Supplementary Fig. S3). Silicon rubber (Ecoflex ${ }^{\mathrm{TM}}$ 00-50) was obtained from Smooth-On, Inc. N-heptane (AR, 98\%) was acquired from Shanghai Aladdin Biochemical Technology Co., Ltd. Ethyl alcohol (AR, $\geq 99.7 \%$ ) was purchased from Sinopharm Chemical Reagent Co., Ltd. Deionized water was used as the substrate to prepare single graphene film and graphene/ Ecoflex Janus film at the air/water interface.

\subsection{Fabrication of Graphene Film}

Firstly, graphene slurry ( $5 \mathrm{~g})$ was dispersed in $250 \mathrm{~mL}$ anhydrous ethanol, followed by strong ultrasonication $(250 \mathrm{~W})$ for about $6 \mathrm{~h}$ to obtain a stable dispersion. Then, the graphene dispersion $(35 \mathrm{~mL})$ was sprayed equably on the water surface. After stabilization for about $30 \mathrm{~min}$, the microporous sponge was put on one side of the water/air interface to siphon water, while the graphene nanosheets were tightly stacked toward the opposite direction of the siphon. Finally, the graphene film with a closely packed structure was formed at the water/air interface until the pre-assembled film could not be compressed further.

\subsection{Fabrication of Graphene/Ecoflex Janus Film}

The Ecoflex part A and part B (1:1, w/w) were dissolved in n-heptane with a weight ratio of $7.71 \%$ and then ultrasonicated for 5 min to obtain a homogeneous solution. After that, the 



Fig. 1 a The lateral line perception system of fish. b The microscopic image of fish scales. c The digital photograph of lateral line canal. d The schematic diagram of a lateral line system. e Schematic illustration of the lateral line. $\mathbf{f}, \mathbf{g}$ The digital photographs of the graphene/Ecoflex Janus film under water at depths of $0,10,20,30,40$, and $50 \mathrm{~cm}$, respectively

Ecoflex solution $(35 \mathrm{~mL})$ was dropwise added onto the graphene/air interface along the container wall, followed by the evaporation of $n$-heptane and curing of Ecoflex at room temperature for $6 \mathrm{~h}$. Subsequently, the graphene/Ecoflex ultrathin Janus hybrid film was obtained at the water/air interface.

\subsection{Preparation of Lateral-Line-Like Underwater Mechanical Sensor}

At first, a hole with an appropriate diameter was cut out in the middle of the petri dish, and then an ultrathin layer of Ecoflex prepolymer was scraped on its surface after rinsing the petri dish with anhydrous ethanol. The prepared graphene/Ecoflex Janus film was transferred onto the surface of the petri dish with part of the film attached to the substrate and the other part self-supported. After the curing of Ecoflex prepolymer layer at $60{ }^{\circ} \mathrm{C}$ for $1 \mathrm{~h}$, the film was closely attached to the substrate. Then, the aluminium wire was connected to the graphene side with the aid of silver paste curing in a $60{ }^{\circ} \mathrm{C}$ oven. After that, another polymethyl methacrylate (PMMA) lid (diameter: $36 \mathrm{~mm}$, height: $15 \mathrm{~mm}$ ) was covered onto the graphene side and sealed with Ecoflex. Finally, the lateralline-like underwater mechanical sensor, whose graphene sensing layer was encapsulated and Ecoflex elastic layer was exposed to water, was successfully fabricated.

\subsection{Characterization and Measurements}

Surface and cross section morphology of the film was observed by Hitachi-S4800 field-emission scanning electron 
microscope (FE-SEM) at an accelerating voltage of $4 \mathrm{kV}$. The microscopic images of fish scales and tapes were captured by an OLYMPUS BX51 polarizing microscope. The Raman scattering measurements were carried out by an R-3000HR spectrometer (Raman Systems, Inc., R-3000 series) excited by a solid-state diode laser $(532 \mathrm{~nm})$, with a frequency range of 3500-200 $\mathrm{cm}^{-1}$. Z1 Zwick/Roell Universal Testing System was used to test the stress-strain characteristics of films. When LUMS detected underwater depth or external stimuli, the electrodes at both ends of the sensor were connected to the electrochemical workstation through wires, and the electrochemical workstation was connected to the computer (Supplementary Fig. S4). Electrochemical Workstation (CH Instruments, CHI660E.Chenhua Co., Shanghai, China) was used to record the real-time current (I) accompanied by a constant voltage $\left(V_{0}\right)$ of $1 \mathrm{~V}$, while the real-time resistance $(R)$ was calculated by the equation $R=V_{0} / I$. The water contact angle was measured using Dataphysics OCA25 instrument by carefully dropping a $3-\mu \mathrm{L}$ water droplet on the surface of film. AFM measurements were conducted using Dimension ICON SPM (Bruker, USA) in a Peak Force tapping mode.

\section{Results and Discussion}

\subsection{Fabrication and Application of the Janus Films}

Inspired by the structure and sensing mechanism of the lateral line, a Janus film composed of graphene and Ecoflex was designed via interfacial functionalization strategy. The assembled graphene film functioned as a sensing layer of the Janus film. It was fabricated via Marangoni effect induced self-assembly and the capillary force driving compression at the air/water interface [32-34]. And the thickness of the assembled graphene film was about $200 \mathrm{~nm}$ (Supplementary Fig. S5). Subsequently, Ecoflex prepolymer elastomer dispersed in heptane solution was gradually dropped onto the surface of graphene film, followed by a curing procedure. The interfacial functionalization strategy enables the formation of ultrathin Janus film at the air/water interface (Supplementary Fig. S6a), and the initial resistance of the Janus film $(59.5 \mu \mathrm{m} \times 1.5 \mathrm{~cm} \times 3.9 \mathrm{~cm})$ was $6.98 \mathrm{k} \Omega$. In order to explore the hydrophilicity and hydrophobicity of the Janus film, water contact angle was measured by carefully dropping a $3-\mu \mathrm{L}$ water droplet on the surface of films. In comparison with the pure graphene film with a water contact angle of $20.2^{\circ}$, the graphene side of the Janus film displayed a bigger water contact angle of $88.9^{\circ}$ (Supplementary Fig. S7). In addition, the water contact angle of Ecoflex side of the Janus film is $109.4^{\circ}$, similar to that of the pure Ecoflex film (Supplementary Fig. S7). The hydrophobic property of Ecoflex layer ensures the hydrophobicity of the Janus film and makes it possible to be used underwater.

Owing to the ultrathin and conductive features of the Janus film, it can be driven to deform by tiny water vibration and low/high water pressure for real-time electrical signal output. As an analogue to the lateral line of fish, it can actively detect the mechanical stimuli from the surrounding environments and sense the change of water pressure decided by the water depth (Fig. 1e). Therefore, by mimicking the structure of the real lateral line, the acquired Janus film can be conformally transferred onto a model fish to achieve a lateral-line-like underwater mechanical sensor (LUMS). In our system, a model with a hollow structure was designed to assemble with the Janus film for a self-supported structure, in which the Ecoflex layer was exposed to water and the graphene layer was sealed in the air chamber of the model. Besides, the electrodes were applied on the surface of the graphene layer to form an LUMS (Fig. 1f). With the increase in water depth, the Janus film can experience a simultaneous deformation, accompanied by a corresponding current change for real-time depth detection. As shown in Fig. 1g, LUMS was located at a series of water depths, such as $0,10,20,30$, and $40 \mathrm{~cm}$. It can be clearly observed that the film can be actuated by the water pressure and actively experience a gradual increase in the degree of deformation. The real-time mechanical deformation is expected to induce the corresponding electrical signal change for efficient water depth detection.

\subsection{Structural Characterization and Properties of the Janus Film}

Since the interface functionalization strategy can enable the formation of an ultrathin and transferrable film, the achieved Janus film can be easily transferred onto diverse targets for conformal and robust adhesion. As shown in Fig. 2a, hollow models with different shapes were employed to assemble with the Janus film for a self-supported one. It is observed that the film can adapt smoothly to various sophisticated shapes (e.g. 
triangle, circle, square, and pentacle). When it functioned as a self-supported one, the film can even support objects such as an iron ball with a weight of $8.34 \mathrm{~g}$. The result suggests that the film can demonstrate robust mechanical strength (Fig. 2b). In addition, the ultrathin Janus film also shows excellent conformal characteristic, which can adapt conformally to complex surfaces with complicated embossment. Here, a fish model with fine structure of scales and fins was used. Figure $2 \mathrm{c}$ represents that the film can spread smoothly along the structured embossment of the fish model, in which the scales and fins can be entirely and clearly observed. Similarly, models such as starfish and seaweed were also selected as targeted substrates, demonstrating a good conformal ability of the film (Supplementary Figs. S8 and S9). Moreover, the interface functionalization method also allows the formation of stable interface between graphene and Ecoflex layer. As shown in Fig. 2d, the adhesive tape was tightly adhered onto the graphene side of the film, followed by a peeling-off operation without visible residues. As shown in Supplementary Fig. S10, although there are some black residues on the surface of the tape, they are unevenly distributed and stand for bits of weak-bonded graphene on the surface. And most of the graphene is embedded in Ecoflex and stays stable on the film.

In order to evidence the advantage of this interface functionalization method, two control samples were fabricated in (a)

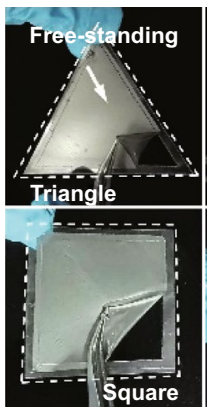

(d)
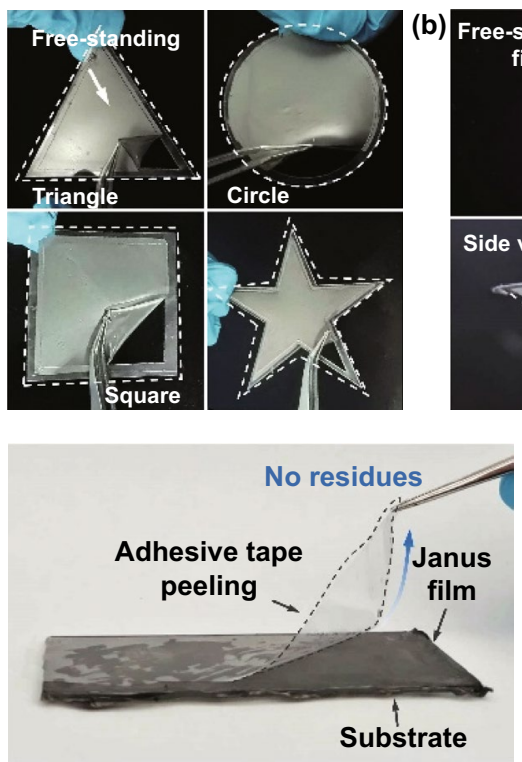

(b) F

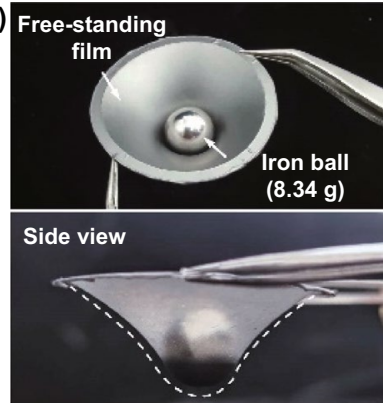

c) (i)
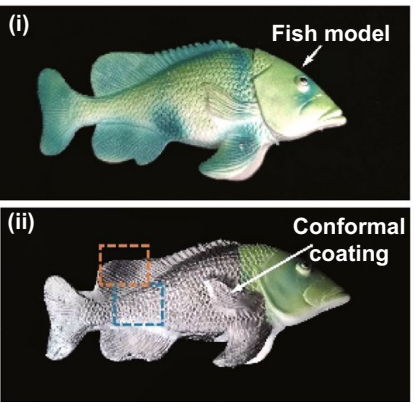

(e)
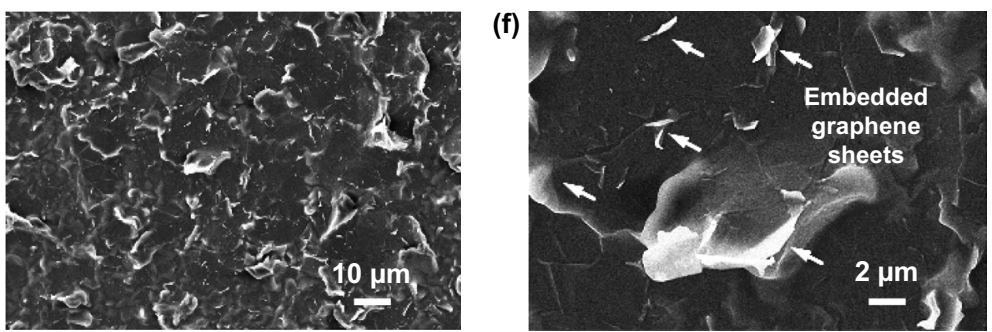

(g)
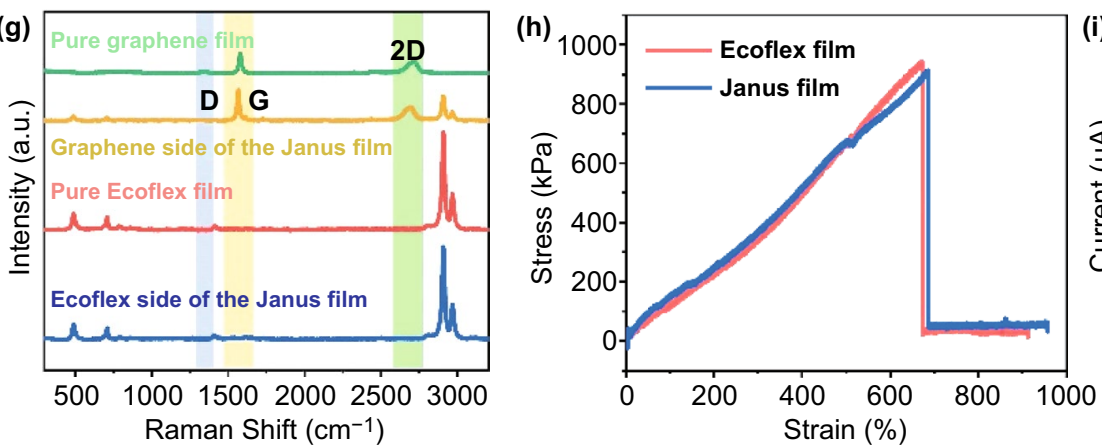

Fig. 2 a The digital photographs of self-supported graphene/Ecoflex Janus film on frames with different shapes. b The digital photographs of the graphene/Ecoflex Janus film holding an iron ball with a weight of $8.34 \mathrm{~g}$. c The digital photographs of the graphene/Ecoflex Janus film attached to the surface of the model fish. $\mathbf{d}$ Peeling-off of the adhesive tape from the graphene side of the Janus film. e, $\mathbf{f}$ SEM images of the graphene side surface of the graphene/Ecoflex Janus film. $g$ Raman spectra of the pure Ecoflex film, pure graphene film and both sides of the graphene/Ecoflex Janus film. h Representative tensile stress-strain curves of the pure Ecoflex film and Janus film. i Current of three kinds of films before and after applying $20 \%$ tensile strain 
our experiments, including the conventional casting method (Supplementary Fig. S6b) and transferring method (Supplementary Fig. S6c). In detail, for the conventional casting method, the assembled graphene film at the water/air interface was transferred onto the glass surface, followed by casting Ecoflex prepolymer solution on its surface. However, the interaction between the graphene film and the glass substrate is too strong to allow for intact peeling-off of the doublelayer film (Supplementary Fig. S11a). For the transferring one, a pure Ecoflex film was firstly fabricated at the water/ air interface, which was further cut and transferred onto the glass substrate. Then, the prepared graphene film was transferred and covered on the Ecoflex film at the air/water interface. After natural air-drying procedure, the doublelayered film was obtained. The results show that the curing Ecoflex film cannot effectively interact with the graphene nanosheets for a Janus film due to the weak penetration to the closely packed sheets. Sample of the transferring method presents poor stability of the graphene layer, which can be easily peeled off using the adhesive tape (Supplementary Fig. S11b). Generally, compared to the conventional casting method and transferring method, the Janus film fabricated by the interface functionalization method demonstrates a stable interface between the graphene layer and the Ecoflex layer. Furthermore, SEM characterization was also conducted to investigate the micro-scale morphology of the Janus film. Figure 2e, f and Supplementary Fig. S12 clearly show that the graphene nanosheets are partially embedded into the elastomeric matrix and the exposed graphene sheets can provide contact sites for the formation of a conductive pathway. And there was only a wrinkled structure of elastomer on the Ecoflex side (Supplementary Fig. S13).

To further investigate the structural information of the Janus film, Raman spectra was also used to characterize the asymmetric structure. As illustrated in Fig. $2 \mathrm{~g}$, the result reveals that the Ecoflex side of Janus film has the same Raman curve as the pure Ecoflex, and the characteristic peak location is mainly in the low frequency $\left(490 \mathrm{~cm}^{-1}, 710 \mathrm{~cm}^{-1}\right)$ and high frequency $\left(2906 \mathrm{~cm}^{-1}\right.$, $2965 \mathrm{~cm}^{-1}$ ) regions. For the graphene side, it not only has the same D $\left(1348 \mathrm{~cm}^{-1}\right), \mathrm{G}\left(1579 \mathrm{~cm}^{-1}\right)$ and $2 \mathrm{D}\left(2718 \mathrm{~cm}^{-1}\right)$ characteristic peaks as the pure graphene film, but also has the same characteristic peaks as the pure Ecoflex. The result further indicates that the side of the graphene layer is partially wrapped by Ecoflex to form a semi-embedded structure, which may result from the infiltration of Ecoflex solution into the graphene layer during the preparation process. In addition, the Raman mapping can also clearly illustrate the Janus structure of this film (Supplementary Fig. S14). More importantly, compared with the pure Ecoflex film, the asymmetric introduction of the graphene layer into the elastomer system may not remarkably affect the mechanical strength (Fig. 2h). Additionally, the electrical information of three samples was also measured in Fig. 2i. When a 20\% strain was applied and then released on the samples, only the current of Janus film can recover to the initial value, representing good electrical stability and repeatability (Supplementary Fig. S15).

Moreover, the strain sensing performance of the Janus film-based strain sensor was investigated, including sensitivity, stability as well as the responses to different strains and stretching frequencies, which was represented by a relative resistance change $\left(\Delta R / R_{0}\right)$ :

$\Delta / R_{0}=\left(R-R_{0}\right) / R_{0}$

where $R$ is the instantaneous resistance at the stretched state and $R_{0}$ is the initial resistance at the relaxed state. The tensile strain $(\varepsilon)$ and Gauge factor (GF) are calculated by Eqs. (2) and (3), respectively:

$\varepsilon=\left(L-L_{0}\right) / L_{0}$

$\mathrm{GF}=\frac{\delta\left(\Delta R / R_{0}\right)}{\delta \varepsilon}$

As shown in Supplementary Fig. S16, with the increase in the concentration of the graphene dispersion, the response of tensile strain range of the Janus film expanded. And the sensitivity of the Janus film gradually decreased in the same tensile strain range. In order to ensure the excellent conductivity and high sensitivity of the Janus film under large deformation, graphene dispersion $\left(1 \mathrm{mg} \mathrm{mL}^{-1}\right)$ was chosen to prepare the assembled graphene film and the Janus film. As shown in Fig. 3a, the Janus film-based strain sensor displays a large GF in the full sensing range. The corresponding GFs for the sensor are 36 ( $\varepsilon: 0-10 \%$ ), 90 ( $\varepsilon: 15-20 \%$ ), and 1070 ( $\varepsilon: 30-35 \%)$, respectively, which demonstrate that the Janus film-based strain sensor has the potential ability to sense external stimuli with high sensitivity. The relative resistance increases with the increasing strain under cyclic tensile strain of $1 \%, 5 \%, 10 \%, 15 \%$, and $20 \%$ (Fig. 3b), which shows good resolution at each different strain. The stability of the Janus film was also studied. As shown in Fig. 3c, the relative resistance change is almost independent of the frequency with a tensile strain of $20 \%$ within a range of 0.1-3 Hz. Besides, the relative resistance change varies 
periodically and no obvious fluctuation or drift occurs in each cycle, presenting excellent stability and long-term durability during cyclic tensile tests between 0 and $20 \%$ tensile strain for 5000 cycles (Fig. 3d).

\subsection{Water Depth Detection of the LUMS}

Based on the sensitive and stable electrical performance of the Janus film, it was further integrated into LUMS to achieve water depth detection. The sensitivity of the conductive material might be negatively affected when assembled into underwater sensors fabricated by polymer encapsulation method. During the polymer encapsulation, the polymer penetrates into the gap of the conductive material, resulting in an increase in the initial resistance of the conductive material. As shown in Supplementary Fig. S17, compared with the sensor achieved by the Janus film encapsulated by Ecoflex, LUMS based on the Janus film possesses good sensing sensitivity and electrical stability. As displayed in Supplementary Fig. S18, a specific model with a hollow circle structure was designed and the Janus film was subsequently transferred onto the model surface with integrated electrodes. Similar to the lateral line of the fish that can sense the water pressure mediated by the pores on the scales, LUMS allows the Ecoflex side exposed to water and the graphene side encapsulated in the chamber. As a result, with the increase in water depth, the generated water pressure can drive the film to deform to a balanced state. Note that the water pressure $\left(P_{1}\right)$ applied on the Janus film was calculated according to Eq. (4):

$P_{1}=\rho g h$

The relationship between gas pressure $\left(P_{2}\right)$ and volume $(V)$ in the covered seal system can be as follows:

$P_{2} V=n R T$

where $\rho$ represents the density of water, $g$ is the acceleration of gravity, and $h$ is the height from LUMS to the water surface, $\mathrm{n}$ is the amount of gas substance, $T$ is the absolute temperature, and $R$ is a constant of about $8.314 \mathrm{~J} \mathrm{~K}^{-1} \mathrm{~mol}^{-1}$. When LUMS was employed to detect water depth, the film experienced inward deformation driven by the water pressure. The film deformation can prominently reduce the volume of the air chamber to further increase the inner air pressure. When $P_{1}$ was in equilibrium with $P_{2}$, the dynamic deformation of the film achieved an equilibrium state.
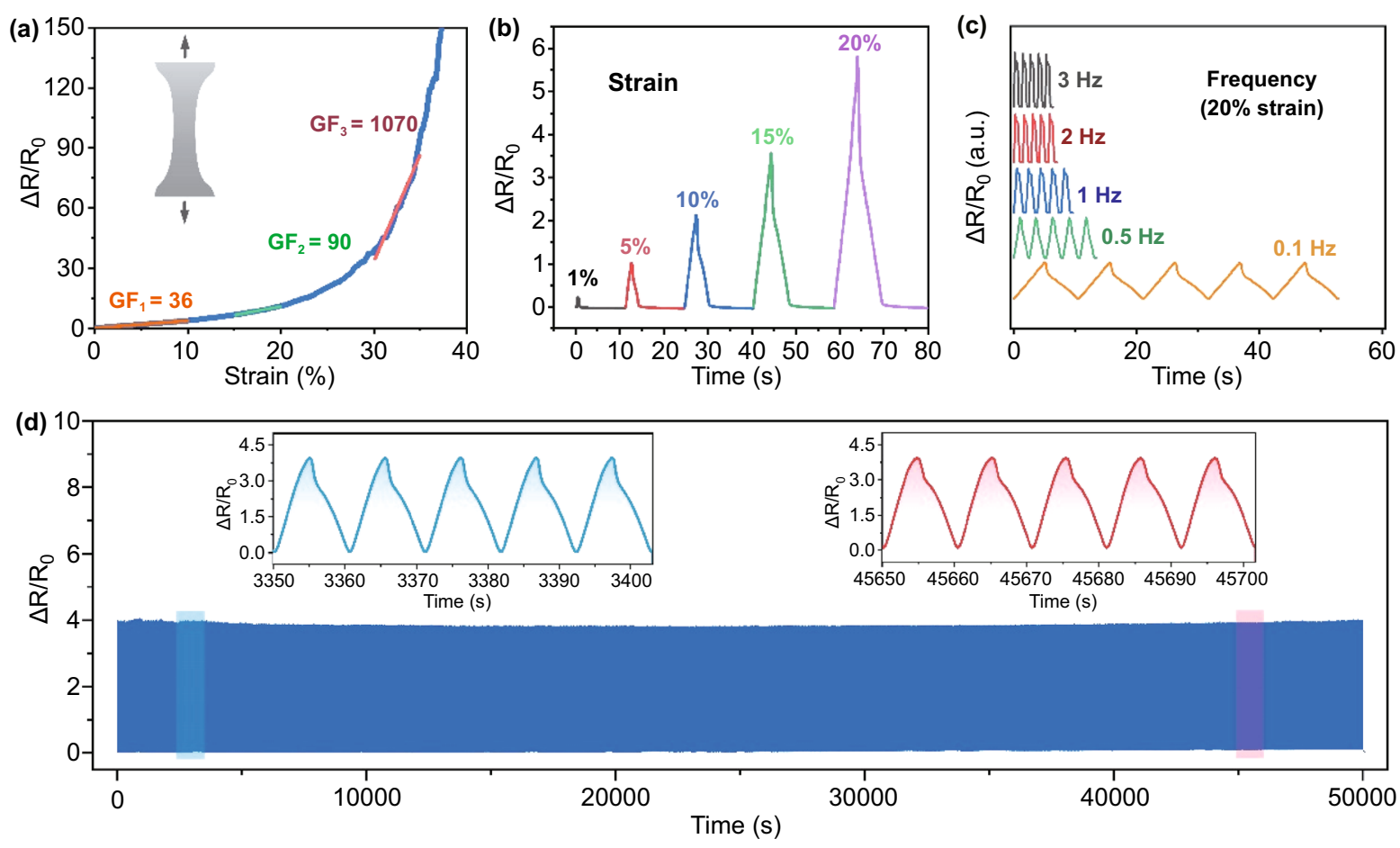

Fig. 3 a The sensing performance of the graphene/Ecoflex Janus film and specific GF values under different tensile strains. $\Delta R / R_{0}$ versus time curve of the Janus film $\mathbf{b}$ under various tensile strains and a stretching velocity of $50 \mathrm{~mm} \mathrm{~min}{ }^{-1}$; $\mathbf{c}$ under a tensile strain of $20 \%$ and various frequencies of $0.1,0.5,1,2$, and $3 \mathrm{~Hz}$; $\mathbf{d}$ under a tensile strain of $20 \%$ for 5000 cycles 
In our system, with the increase in the water depth, there appeared apparent film deformation and simultaneous terraced current platform located at a certain depth. Take the sample of the self-supported film with a diameter of $10 \mathrm{~mm}$ as an example, LUMS can respond sensitively to the depth of $3 \mathrm{~cm}$ and detect up to the depth of $50 \mathrm{~cm}$ (Fig. 4a). When further increasing the depth to $60 \mathrm{~cm}$, the resulted current no more changed and the resultant maximum depth was finally achieved (Supplementary Fig. S19). More interestingly, it is found that the degree of deformation at the same depth is also related to the diameter of the self-supported film. When increasing the diameter of the hollow circle, the degree of deformation may decrease gradually. Thus, devices with four kinds of diameter were designed, including 10, 15, 20, and $25 \mathrm{~mm}$. As illustrated in Fig. 4b-d, all the three samples with certain diameters demonstrate step-like change in current with the increase in water depth. It is found that the maximum detection depth is positively related to the diameter. For example, the sample with a diameter of $25 \mathrm{~mm}$ can realize a good electrical response at a depth up to $1.8 \mathrm{~m}$. However, owing to the decreased film deformation at a certain depth, the relative resistance of these samples experienced a reduced tendency at the same depth with the increase in diameters (Fig. 4e). Furthermore, Fig. 4f and Supplementary Fig. S20 clearly show the positive linear relationship between diameter and maximum water depth.
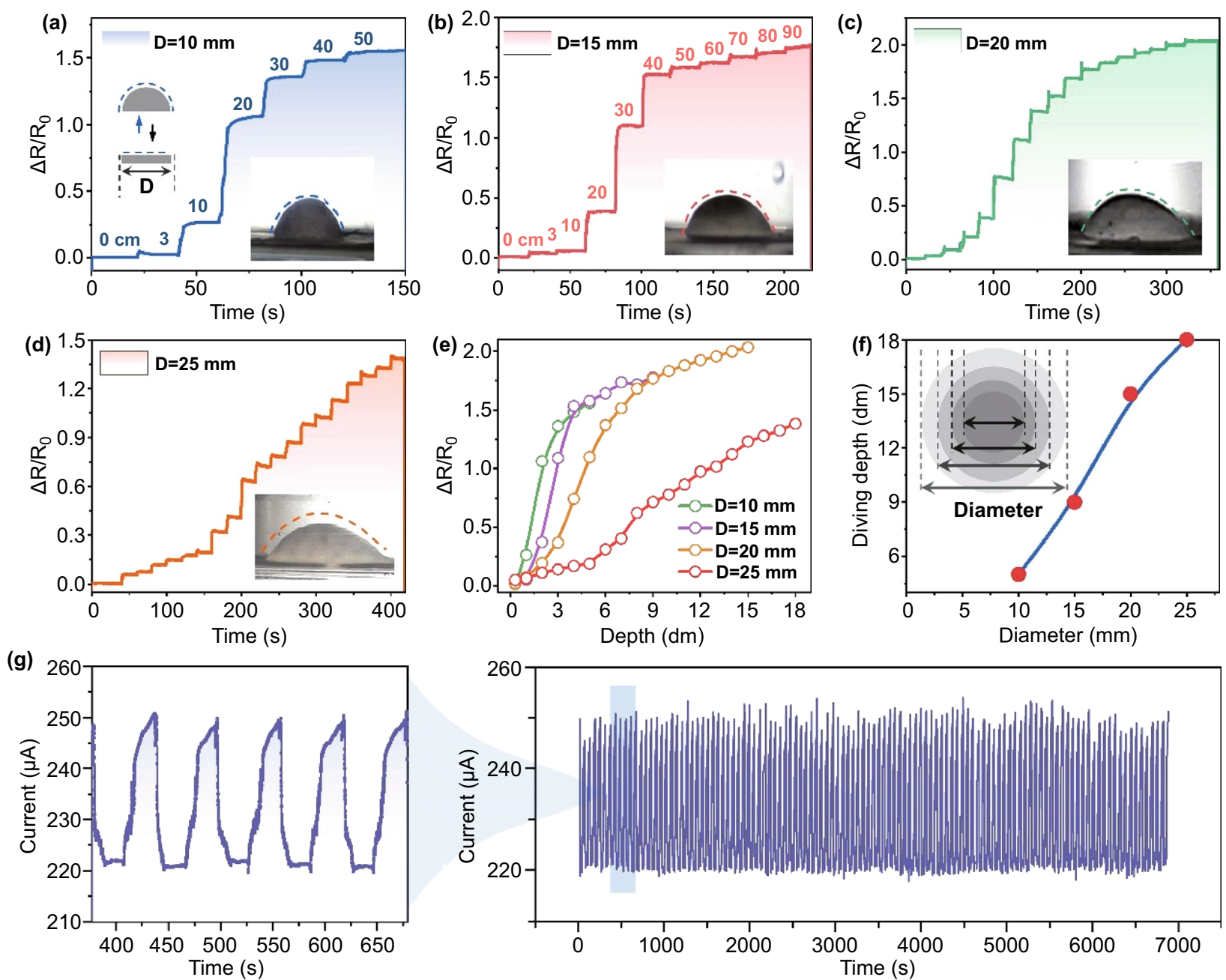

Fig. $4 \Delta R / R_{0}$ versus time curve of LUMS at different depths with the diameter of a $10 \mathrm{~mm}, \mathbf{b} 15 \mathrm{~mm}, \mathbf{c} 20 \mathrm{~mm}$, and d $25 \mathrm{~mm}$, and digital photographs of the Janus film inside LUMS at corresponding maximum measurable depth. e $\Delta R / R_{0}$ versus depth curves of LUMS with different diameters. $\mathbf{f}$ The curve of the maximum measurable depth as a function of the film diameters. $\mathbf{g}$ Output current versus time curve where LUMS went back and forth between the liquid level and the maximum measurable depth 
To further explore the influence of film diameter on sensing performance of LUMS, the response time of sensors of different sizes to the same stimulus was detected. The response time is defined as the time required to reach a relative resistance variation that is equal to $90 \%$ of the peak value. As shown in Supplementary Fig. S21, the response time of the sensor with a film diameter of $10,15,20$, and $25 \mathrm{~mm}$ was $0.032,0.046$, 0.050 , and $0.076 \mathrm{~s}$, respectively. In general, the response time of the sensor increases with the increase of the film diameter. Moreover, repeatability test was also conducted, where the device was moved back and forth between the liquid level and the maximum measurable depth and demonstrated good stability of the electrical performance (Fig. 4g). Considering that LUMS was used for underwater sensing, the influence of water temperature on the sensor's sensing performance was studied. As shown in Supplementary Fig. S22, when the temperature suddenly increased by $3{ }^{\circ} \mathrm{C}$, the relative resistance change kept increasing, and it still could not reach a stable state even after $200 \mathrm{~s}$, which differed greatly from the response time when LUMS was subjected to external stimuli. Therefore, when detecting water depth or perceiving external stimuli, the effect of the water temperature change on the sensing performance of LUMS was almost negligible.

\subsection{Multifunctional LUMS for Underwater and Waterside Monitoring}

It is well known that the fish can not only sense the water depth, but sensitively capture the signal over the water, to avoid some potential dangers. Similarly, LUMS was further employed to sense the mechanical stimuli from the external environment. As revealed in Fig. 5a, LUMS with a diameter of $10 \mathrm{~mm}$ was placed into water to perceive (a)

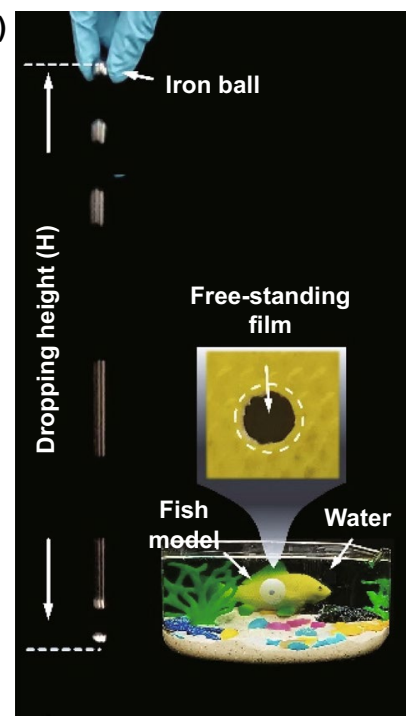

(e)

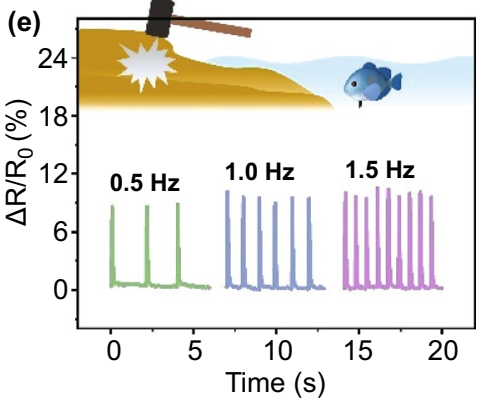

(b) 10

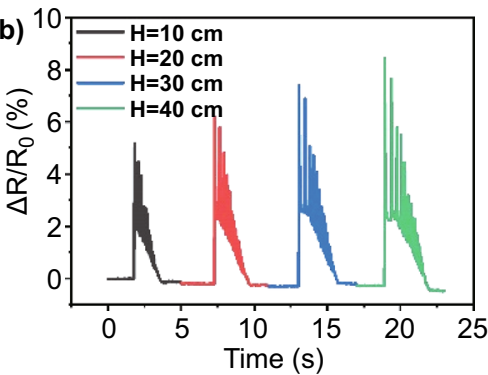

(d) 10

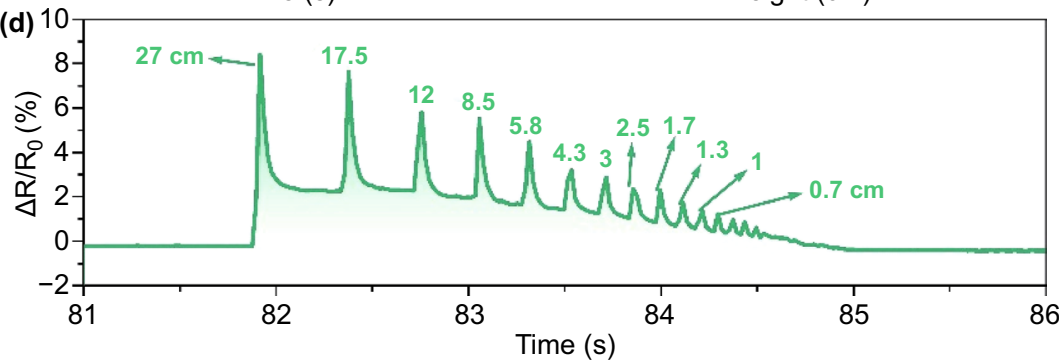

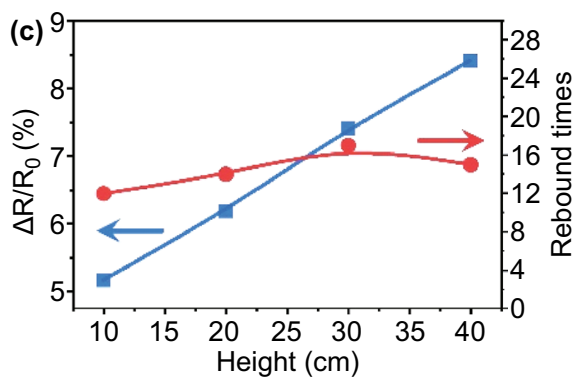

(g) 1

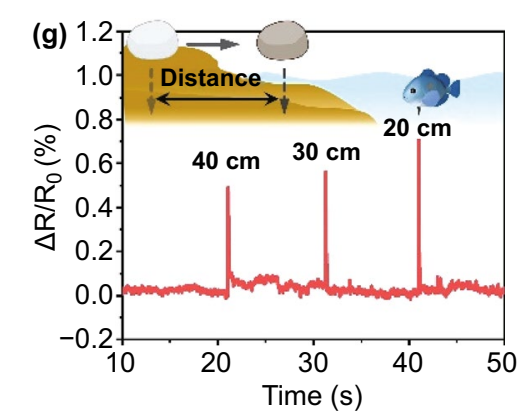

Fig. 5 a Illustration of LMUS to sense the surrounding environment. b $\Delta R / R_{0}$ versus time curve of LUMS stimulated by the steel ball falling from different heights. $\mathrm{c} \Delta R / R_{0}$ versus height curve of LUMS stimulated by steel ball (blue) and the rebound times of steel ball versus height curve (red). $\mathbf{d} \Delta R / R_{0}$ versus time curve of LMUS during the rebounding process of steel ball falling from $40 \mathrm{~cm}$. $\Delta R / R_{0}$ versus time curve of LMUS stimulated $\mathbf{e}$ by percussion at different frequencies; $\mathbf{f}$ by stomping; $\mathbf{g}$ by falling stones with a fixed height but different distances away from the water 
the environment signal. When an iron ball fell from the height of 10, 20, 30, and $40 \mathrm{~cm}$, respectively (Fig. 5b), the device could clearly record the process that the ball hit and bounced off the table before coming to rest on the table. Moreover, the relative resistance was almost linearly related to the falling heights of the iron ball (Fig. 5c), and rebound times was precisely distinguished by LUMS, which was consistent with that counted by video (Fig. 5c). In addition, the time interval between each peak could be used to infer the bounce height of the iron ball (Fig. 5d). When LUMS was placed at a distance of 0,2 , and $4 \mathrm{~cm}$ below the water surface, the maximum relative resistance changes detected by the sensor were $0.05361,0.11281$, and 0.14284 , respectively (Supplementary Fig. S23). But the response time of the sensor showed no significant difference with the change of underwater position. In general, the intensity of the relative resistance change generated by mechanical vibration increased as the depth of LUMS increased underwater. Besides, LUMS could detect signals with different frequencies produced by hitting the ground with a hammer (Fig. 5e) as well as the signal produced by stomping (Fig. 5f). Tests were also carried out to evaluate LUMS's perception of objects falling at different distances $(20,30$, and $40 \mathrm{~cm})$ away from the water (Fig. 5g), where the relative resistance decreased with the increase of the distance, demonstrating the good sensitivity. Therefore, our well-designed LUMS is expected to demonstrate potential applications in underwater and waterside monitoring.

To further simulate the excellent capability of underwater perception of fish, analogous to the function of the lateral line, LUMS can also sensitively capture the tiny and intense mechanical stimuli from and beneath the water surface. For instance, when the simulated wind with varied velocities was applied on the water surface, LUMS could rapidly perceive the fluctuation induced by the wind. Note that there are remarkable differences in the normalized resistance between the velocity of 3.4 and $4.2 \mathrm{~m} \mathrm{~s}^{-1}$ (Fig. 6a). In addition, a water droplet from different heights can also be sensitively detected. As shown in Fig. $6 \mathrm{~b}$, the droplet fallen from the height of $20,40,60 \mathrm{~cm}$ above the water surface could be effectively recognized. Moreover, in addition to the single water droplet, the raining condition was also simulated in our system. Figure $6 \mathrm{c}$ illustrates that LUMS can regularly respond to the simulated raining with different intensities, including 60, 144,

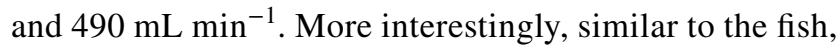
LUMS can actively perceive the water fluctuation induced by the fallen fishhook (Fig. 6d). In addition, some natural fallen objects such as leaf, branch, and stone, which led to tiny or drastic vibration/fluctuation of water surface, could be clearly detected (Fig. 6e). Furthermore, to efficiently tackle the potential danger beneath water surface, the sensor also needs to realize a sensitive perception of the underwater stimuli. As a proof of concept, a robotic shark swinging its tail was used to move around the sensor. As a result, LUMS could effectively monitor the distinct movement states of shark tails with low or large amplitudes, demonstrating significant potentials in applications of underwater monitoring (Fig. 6f).

\section{Conclusions}

In summary, enlightened by the fish sensing system, an artificial fish lateral line enabled by the graphene-based thin film was designed to simultaneously detect wide-range water depth and sensitively perceive tiny vibration. The Janusstructured graphene/Ecoflex ultrathin film was endowed with conformal, elastic, conductive and deformable properties, which could adapt smoothly to the structured surface. When integrated to be a self-supported sensor, it can achieve an efficient and stable detection of water depth up to $1.8 \mathrm{~m}$. Furthermore, tiny vibration (e.g. wind blowing, raining, leaf falling, and underwater attacking) from water surface or underwater can also be sensitively captured. The bioinspired Janus film-enabled underwater sensor shows significant potentials in the field of water depth detection and vibration perception in the water environment. 

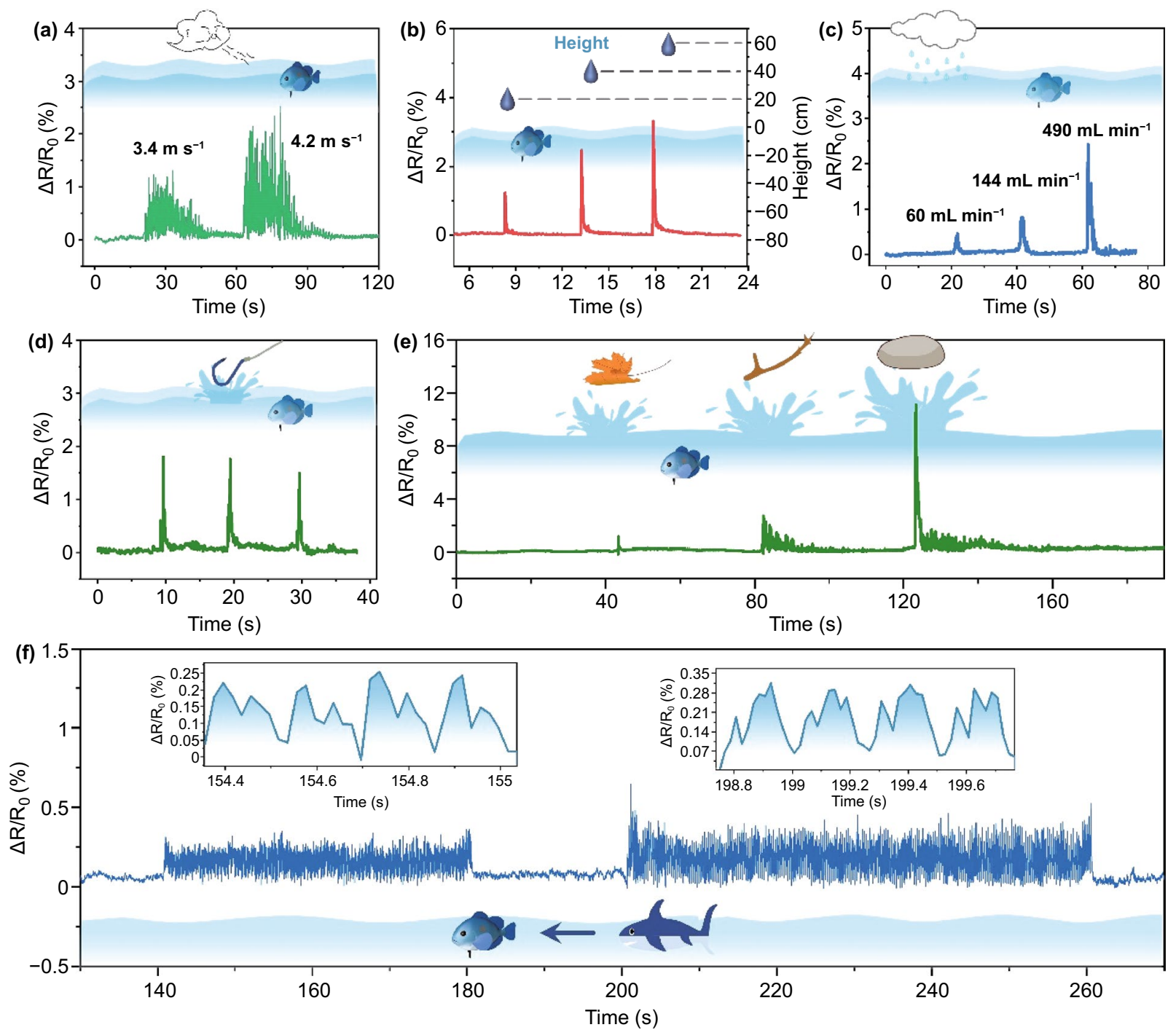

Fig. $6 \Delta R / R_{0}$ versus height curve of LUMS stimulated a by different wind speeds disturbing the liquid level and $\mathbf{b}$ by water droplet falling from different heights. $\Delta R / R_{0}$ versus height curve of LUMS stimulated $\mathbf{c}$ by different rainfalls; $\mathbf{d}$ by falling fishhook; $\mathbf{e}$ by a falling leaf, branch and stone; $\mathbf{f}$ by different fish swing amplitudes

Acknowledgements This work was supported by the Natural Science Foundation of China (51803226, 52073295), the SinoGerman Mobility Program (M-0424), Key Research Program of Frontier Sciences, Chinese Academy of Sciences (QYZDBSSWSLH036), Bureau of International Cooperation, Chinese Academy of Sciences (174433KYSB20170061), Ningbo Science and Technology Bureau (2021Z127), and K. C. Wong Education Foundation (GJTD-2019-13).
Funding Open access funding provided by Shanghai Jiao Tong University.

Open Access This article is licensed under a Creative Commons Attribution 4.0 International License, which permits use, sharing, adaptation, distribution and reproduction in any medium or format, as long as you give appropriate credit to the original author(s) and the source, provide a link to the Creative Commons licence, and 
indicate if changes were made. The images or other third party material in this article are included in the article's Creative Commons licence, unless indicated otherwise in a credit line to the material. If material is not included in the article's Creative Commons licence and your intended use is not permitted by statutory regulation or exceeds the permitted use, you will need to obtain permission directly from the copyright holder. To view a copy of this licence, visit http://creativecommons.org/licenses/by/4.0/.

Supplementary Information The online version contains supplementary material available at https://doi.org/10.1007/ s40820-022-00799-4.

\section{References}

1. Y.F. Hu, J. Yang, Q.S. Jing, S.M. Niu, W.Z. Wu et al., Triboelectric nanogenerator built on suspended 3D spiral structure as vibration and positioning sensor and wave energy harvester. ACS Nano 7(11), 10424-10432 (2013). https://doi.org/10. 1021/nn405209u

2. N.J. Lindsey, T.C. Dawe, J.B. Ajo-Franklin, Illuminating seafloor faults and ocean dynamics with dark fiber distributed acoustic sensing. Science 366(6469), 1103-1107 (2019). https://doi.org/10.1126/science.aay5881

3. Y. Zou, P.C. Tan, B.J. Shi, H. Ouyang, D.J. Jiang et al., A bionic stretchable nanogenerator for underwater sensing and energy harvesting. Nat. Commun. 10, 2695 (2019). https://doi. org/10.1038/s41467-019-10433-4

4. Y. Gao, J.F. Song, S.M. Li, C. Elowsky, Y. Zhou et al., Hydrogel microphones for stealthy underwater listening. Nat. Commun. 7, 12316 (2016). https://doi.org/10.1038/ncomms12316

5. S.C. Li, J.L. Zheng, J. Yan, Z.J. Wu, Q. Zhou et al., Gate-free hydrogel-graphene transistors as underwater microphones. ACS Appl. Mater. Interfaces 10(49), 42573-42582 (2018). https://doi.org/10.1021/acsami.8b14034

6. Z.P. Yang, Y.T. Niu, S. Wang, Y.Y. Zhang, Z.Z. Yong et al., Rational design of fast recoverable shape-memory photoelectric spring in response to tiny deformation for monitoring underwater microvibration. Compos. Part B Eng. 202, 108402 (2020). https://doi.org/10.1016/j.compositesb.2020.108402

7. S. Wang, T. Zhang, K.W. Li, S.Y. Ma, M. Chen et al., Flexible piezoelectric fibers for acoustic sensing and positioning. Adv. Electron. Mater. 3(3), 1600449 (2017). https://doi.org/ 10.1002/aelm.201600449

8. L.P. Liu, Z.B. Jiao, J.Q. Zhang, Y.C. Wang, C.C. Zhang et al., Bioinspired, superhydrophobic, and paper-based strain sensors for wearable and underwater applications. ACS Appl. Mater. Interfaces 13(1), 1967-1978 (2021). https://doi.org/10.1021/ acsami.0c18818

9. Z.C. Yu, P.Y. Wu, Underwater communication and optical camouflage ionogels. Adv. Mater. 33(24), 2008479 (2021). https://doi.org/10.1002/adma.202008479
10. D. Kim, J. Yoon, Water-borne fabrication of stretchable and durable microfibers for high-performance underwater strain sensors. ACS Appl. Mater. Interfaces 12(18), 20965-20972 (2020). https://doi.org/10.1021/acsami.0c04013

11. N. Li, L.F. Qiao, J.T. He, S.X. Wang, L.M. Yu et al., Solardriven interfacial evaporation and self-powered water wave detection based on an all-cellulose monolithic design. Adv. Funct. Mater. 31(7), 2008681 (2020). https://doi.org/10.1002/ adfm.202008681

12. C. Wang, B. Zhang, Y. Li, X.N. Zhao, Suspended graphene hydroacoustic sensor for broadband underwater wireless communications. IEEE Wirel. Commun. 27(5), 44-52 (2020). https://doi.org/10.1109/mwc.001.2000056

13. S.A. Moshizi, S. Azadi, A. Belford, A. Razmjou, S.Y. Wu et al., Development of an ultra-sensitive and flexible piezoresistive flow sensor using vertical graphene nanosheets. Nano-Micro Lett. 12, 109 (2020). https://doi.org/10.1007/ s40820-020-00446-w

14. S. Prabhu, A.B. Pai, G.S. Arora, M.R. Kusshal, V. Pandin et al., Design of piezo-resistive type acoustic vector sensor using graphene for underwater applications. IOP Conf. Ser. Mater. Sci. Eng. 1045, 012015 (2021). https://doi.org/10.1088/ 1757-899x/1045/1/012015

15. R.X. Xu, K.L. Zhang, X.Y. Xu, M.H. He, F.C. Lu et al., Superhydrophobic $\mathrm{WS}_{2}$-nanosheet-wrapped sponges for underwater detection of tiny vibration. Adv. Sci. 5(4), 1700655 (2018). https://doi.org/10.1002/advs.201700655

16. M.M. Zhang, S.L. Fang, J. Nie, P. Fei, A.E. Aliev et al., Selfpowered, electrochemical carbon nanotube pressure sensors for wave monitoring. Adv. Funct. Mater. 30(42), 2004564 (2020). https://doi.org/10.1002/adfm.202004564

17. S.H. Kwon, J. Park, W.K. Kim, Y.J. Yang, E. Lee et al., The effective energy harvesting method from natural water motion active transducer. Energy Environ. Sci. 7(10), 3279-3283 (2014). https://doi.org/10.1039/c4ee00588k

18. N.R. Alluri, B. Saravanakumar, S.J. Kim, Flexible, hybrid piezoelectric film $\left(\mathrm{BaTi}_{(1-x)} \mathrm{Zr}_{(x)} \mathrm{O}_{3}\right) / \mathrm{PVDF}$ nanogenerator as a self-powered fluid velocity sensor. ACS Appl. Mater. Interfaces 7(18), 9831-9840 (2015).

19. X.J. Su, H.Q. Li, X.J. Lai, Z.H. Chen, X.R. Zeng, 3D porous superhydrophobic CNT/EVA composites for recoverable shape reconfiguration and underwater vibration detection. Adv. Funct. Mater. 29(24), 1900554 (2019). https://doi.org/ 10.1002/adfm.201900554

20. Y.M. Ni, J.Y. Huang, S.H. Li, X.Q. Wang, L.X. Liu et al., Underwater, multifunctional superhydrophobic sensor for human motion detection. ACS Appl. Mater. Interfaces 13(3), 4740-4749 (2021). https://doi.org/10.1021/acsami.0c19704

21. S.N. Lai, C.K. Chang, C.S. Yang, C.W. Su, C.M. Leu et al., Ultrasensitivity of self-powered wireless triboelectric vibration sensor for operating in underwater environment based on surface functionalization of rice husks. Nano Energy $\mathbf{6 0}$, 715-723 (2019). https://doi.org/10.1016/j.nanoen.2019.03. 067 
22. G.M. Ding, W.C. Jiao, R.G. Wang, Z.M. Chu, Y.F. Huang, An underwater, self-sensing, conductive composite coating with controllable wettability and adhesion behavior. J. Mater. Chem. A 7(19), 12333-12342 (2019). https://doi.org/10.1039/ c9ta02691f

23. T. Wang, Y. Si, S. Luo, Z. Dong, L. Jiang, Wettability manipulation of overflow behavior via vesicle surfactant for waterproof surface cleaning. Mater. Horiz. 6(2), 294-301 (2019). https://doi.org/10.1039/c8mh01343h

24. Y. Liang, F. Ni, L. Zhang, T. Zhang, S. Wang et al., Biomimetic underwater self-perceptive actuating soft system based on highly compliant, morphable and conductive sandwiched thin films. Nano Energy 81, 105617 (2021). https://doi.org/10. 1016/j.nanoen.2020.105617

25. G.M. Ding, W.C. Jiao, R.G. Wang, M.L. Yan, Z.M. Chu et al., Superhydrophobic heterogeneous graphene networks with controllable adhesion behavior for detecting multiple underwater motions. J. Mater. Chem. A 7(30), 17766-17774 (2019). https://doi.org/10.1039/c9ta04648h

26. S.B. Ji, C.J. Wan, T. Wang, Q.S. Li, G. Chen et al., Waterresistant conformal hybrid electrodes for aquatic endurable electrocardiographic monitoring. Adv. Mater. 32(26), 2001496 (2020). https://doi.org/10.1002/adma.202001496

27. J.C. Montgomery, J.A. Macdonald, Sensory tuning of lateral line receptors in antarctic fish to the movements of planktonic prey. Science 235(4785), 195-196 (1987). https://doi.org/10. 1126/science.235.4785.195
28. J. Montgomery, S. Coombs, M. Halstead, Biology of the mechanosensory lateral line in fishes. Rev. Fish Biol. Fisher. 5, 399-416 (1995). https://doi.org/10.1007/bf01103813

29. S.W. Chou, Z.W. Chen, S.Y. Zhu, R.W. Davis, J.Q. Hu et al., A molecular basis for water motion detection by the mechanosensory lateral line of zebrafish. Nat. Commun. 8, 2234 (2017). https://doi.org/10.1038/s41467-017-01604-2

30. R. Winklbauer, development of the lateral line system in Xenopus. Prog. Neurobiol. 32(3), 181-206 (1989). https://doi.org/ 10.1016/0301-0082(89)90016-6

31. X.W. Zheng, W. Wang, M.L. Xiong, G.M. Xie, Online state estimation of a fin-actuated underwater robot using artificial lateral line system. IEEE Trans. Robot. 36(2), 472-487 (2020). https://doi.org/10.1109/tro.2019.2956343

32. P. Xiao, J.C. Gu, C.J. Wan, S. Wang, J. He et al., ultrafast formation of free-standing 2D carbon nanotube thin films through capillary force driving compression on an air/water interface. Chem. Mater. 28(19), 7125-7133 (2016). https://doi.org/10. 1021/acs.chemmater.6b03420

33. X.M. Li, T.T. Yang, Y. Yang, J. Zhu, L. Li et al., Large-area ultrathin graphene films by single-step marangoni self-assembly for highly sensitive strain sensing application. Adv. Funct. Mater. 26(9), 1322-1329 (2016). https://doi.org/10.1002/ adfm.201504717

34. M. Li, H. Zhang, M. Liu, B. Dong, A light-powered shapeconfigurable micromachine. Mater. Horiz. 5(3), 436-443 (2018). https://doi.org/10.1039/c7mh00968b 